\title{
ANAL CANCER
}

\author{
D J Jones, R D James
}

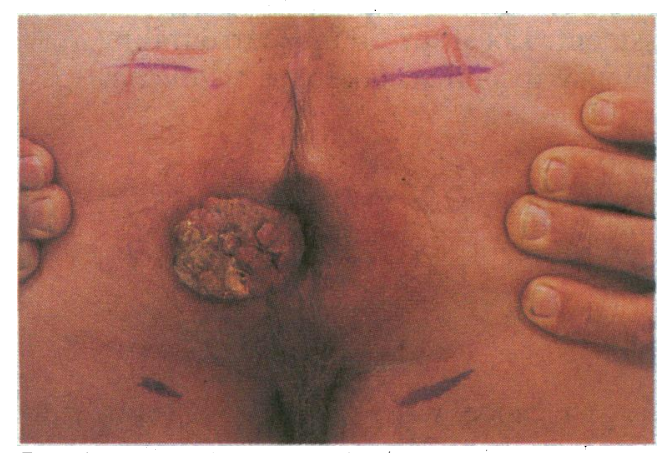

Proliferative anal cancer.

\section{Pathology}

\section{Types of anal neoplasm}

- Squamous cell carcinoma

- Malignant melanoma

- Lymphoma

- Anal gland adenocarcinoma

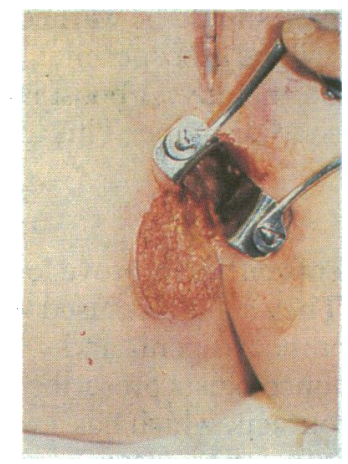

Ulcerating cancer of the anal margin.
Anal cancer is relatively uncommon, accounting for about $4 \%$ of anorectal malignancies. The condition is of increasing interest owing to appreciable changes in the patterns of treatment. The traditional treatment for anal cancer was a radical abdominoperineal excision, leaving the patient with a permanent colostomy. Emerging evidence shows that most anal cancers respond to a combination of radiotherapy and chemotherapy, which improves survival and enables radical surgery to be avoided.

About $80 \%$ of anal malignancies are squamous cell carcinomas. They show a range of histological features, varying from predominantly well differentiated keratinising large cell tumours at the anal margin to less differentiated non-keratinising small cell carcinomas in the upper anal canal. The precise histological pattern is rarely important, but their position in the anus influences clinical decisions.

Anal canal carcinomas arise at or above the dentate line and are not visible at the anus.

Anal margin cancers occur below the dentate line and are visible; they behave like basal cell skin carcinomas and have a more favourable prognosis than anal canal cancers. If advanced the position of origin is difficult to determine.

Anal carcinomas appear as ulcers or proliferative growths with ulcerated areas. They become hard and fixed as the malignancy progresses. They spread directly to invade the underlying anal sphincters, which are affected in three quarters of patients at presentation. Tumours above the dentate line spread to the superior haemorrhoidal lymph nodes while those below the dentate line metastasise to the inguinal lymph nodes.

There is an increased incidence of anal cancer among male homosexuals, people who practice anal intercourse, and those with a history of genital warts. This knowledge prompted a search for a sexually transmitted agent which may be implicated in the pathogenesis of anal cancer. A corollary has been drawn with cervical cancer and its association with human papillomavirus infection. A half of patients with anal cancer have human papillomavirus types 16 and 18 DNA incorporated in the genome of their tumour cells. Some patients with genital warts have evidence of intraepithelial neoplasia around the anus, analogous to cervical intraepithelial neoplasia, which is recognised to be a premalignant condition. Though some of this evidence may be circumstantial, there is growing concern that the increasing incidence of genital human papillomavirus infection will ultimately lead to an increased incidence of anal cancer.
Perianal papillomas are increasing in incidence and may predispose to anal cancer. 


\section{Clinical features}

\section{Staging of anal cancers}

Anal canal modified (Papillon, 1982)

T1 $<2 \mathrm{~cm}$

T2 $2-5 \mathrm{~cm}$

T3 $>5 \mathrm{~cm}$

T4a Invading vaginal mucosa

T4b Invading surrounding structures other than skin, rectum, or vagina

Tx Insufficient information available

Anal margin

T1 $<2 \mathrm{~cm}$

T2 $2-5 \mathrm{~cm}$

T3 $>5 \mathrm{~cm}$

T4 Extension to muscle, bone, etc
Overall anal cancers are more common in women, although lesions at the anal margin are more common in men. Most patients are in their 50 s or $60 \mathrm{~s}$. They present with bleeding, pain, swelling, and ulceration around the anus. As the tumour advances patients suffer worsening pain, disturbed bowel habit, incontinence, and rectovaginal fistulas. Many patients have enlarged inguinal lymph nodes, but only $50 \%$ of palpable glands contain tumour.

Early cancers may be confused with papillomas, warts, fissures, and haemorrhoids, which may lead to delay in diagnosis and treatment. Clinical examination under anaesthesia is usually necessary as local tenderness often prevents thorough assessment. Local spread into surrounding structures is determined and biopsy specimens taken for histological confirmation and classification.

The traditional treatment for anal cancer was a radical abdominoperineal resection and a permanent colostomy. In 1974 an American report suggested that patients with anal cancer could be cured by a combination of 5-fluorouracil and mitomycin. At the same time there were reports from France and the United Kingdom on the curative effects of radiotherapy. Within a few years primary surgical treatment was replaced with radiotherapy with concomitant chemotherapy. A current British trial is designed to assess whether the drugs are really necessary. In the meantime all patients with anal cancer should be assessed jointly by a radiotherapist and a surgeon. The vast majority have a trial of radical radiotherapy, with or without chemotherapy, and surgery should be held in reserve for cases in which this fails.

The initial radiotherapy field includes the tumour and the inguinal lymph nodes. The patient is treated over 4-5 weeks. This inevitably gives rise to moist desquamation of the perineum. Young women should be warned that they will suffer an artificial menopause and men will be azoospermic. The skin heals three weeks after the completion of radiotherapy. A boost is generally given to the primary tumour by using a radioisotope. This requires a short general anaesthetic.

It is not proved that concomitant cytotoxic agents either improve local control or reduce the risk of distant metastases. They should be used with caution in elderly people. Their main risk is thrombocytopenia and agranulocytosis caused by mitomycin, and most oncologists prescribe antibiotic prophylaxis (co-trimoxazole). 5-Fluorouracil, which has a very short biological half life, must be given by slow infusion to ensure synergy with radiation.

The rate of cure for radiotherapy is at least $50 \%$, but many failures of treatment of local tumours can be salvaged by abdominoperineal resection. Aggressive tumours metastasise to the liver and occasionally to the bones, abdominal lymph nodes, or even the brain. Chemotherapy in these patients can often produce a remission of several months.

\section{Surgery}

Radical surgery is now reserved for patients who fail to respond to chemoradiation and patients with obstructive cancers who benefit from a colostomy while they undergo treatment. Surgery is also indicated for small cancers at the anal margin which are less than $2 \mathrm{~cm}$ in diameter and have not invaded the anal sphincter. They can be excised locally, but less than $5 \%$ of anal cancers fulfil these criteria. 


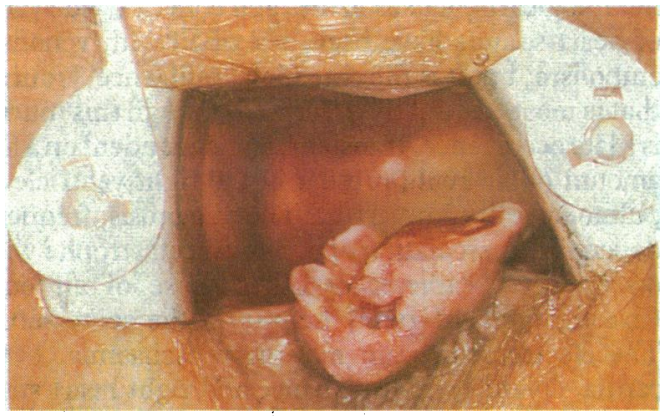

Tumours $<5 \mathrm{~cm}$ in diameter and at the anal margin have a more favourable prognosis.

\section{Prognosis}

For prognostic purposes most information of clinical value is obtained by distinguishing tumours arising at the anal margin from those in the anal canal and separating them into those greater or less than $5 \mathrm{~cm}$ in diameter. The tumour is relatively uncommon so there have been relatively few large follow up studies, but the five year survival rate is about $50 \%$.

The photographs were prepared by the department of medical illustration, Christie Hospital and Holt Radium Institute. The full anal UKCCR trial protocol is available from the CRC Clinical Trial Centre, Rayne Institute, London.

$\mathrm{Mr} \mathrm{D}$ J Jones is lecturer and honorary senior registrar, department of surgery, Hope Hospital, Salford, and Dr R D James is consultant radiotherapist, Christie Hospital and Holt Radium Institute, Manchester.

The ABC of Colorectal Diseases has been edited by Mr D J Jones and Professor M H Irving, Hope Hospital, Salford.

\title{
Venous air embolism associated with removal of central venous catheter
}

\author{
Peter Mennim, Cormac F Coyle, John D Taylor
}

Air embolism may occur not only when inserting and manipulating central venous catheters but also when removing them.
Leighton Hospital, Crewe CW1 4QJ

Peter Mennim, senior medical house officer

Cormac F Coyle, medical registrar

John D Taylor, surgical registrar

Correspondence to: Mr J D Taylor, Transplant Unit, Royal Liverpool University Hospital, Liverpool L7 8XP.
The need to avoid air embolism when inserting and manipulating central venous catheters is widely appreciated but the risk after catheter removal is less widely known. This has implications regarding the delegation of practical procedures. ${ }^{1}$

\section{Case report}

A 73 year old man was admitted for elective repair of an $8 \mathrm{~cm}$ abdominal aortic aneurysm. The past history included a myocardial infarction complicated by ventricular fibrillation eight years previously and an episode of acute left ventricular failure one year before when mitral incompetence was noted. The preoperative echocardiogram showed minimal mitral regurgitation and an ejection fraction of $54 \%$. The aneurysm repair was uneventful, lasting 90 minutes. An episode of pulmonary oedema on the first day was easily treated with diuretics and oxygen. Thereafter, he had an uneventful course, returning from the intensive care unit to the ward on the second postoperative day.

A pulmonary flotation catheter $(7 \cdot 5$ French gauge) had been inserted into the right internal jugular vein at the time of operation and the catheter sheath was removed by the houseman on the fifth day. About two minutes later the patient collapsed and became unresponsive with no palpable cardiac output. Cardiac massage was begun. On arrival the arrest team noted a spontaneous respiration rate of $18 / \mathrm{min}$ with good ventilatory efforts. Pulse was $120 / \mathrm{min}$, regular, and in sinus rhythm. Blood pressure was $90 / 60 \mathrm{~mm} \mathrm{Hg}$ and there was good colour and no cyanosis. The patient was sweating profusely. He was unresponsive to painful stimuli. Pupils were of normal size and reacting symmetrically. There were no focal neurological signs. Chest sounds were normal but examination of the heart disclosed widespread loud, harsh, crunching sounds throughout systole and diastole over the whole precordium. The originally recorded murmur of mitral incompetence could not be heard.

Examination of abdomen and legs showed nothing abnormal apart from the recent incision. Blood glucose concentration was $6.7 \mathrm{mmol} / \mathrm{l}$, and haemoglobin and

\section{Prevention}

Central venous catheters must be removed with the patient supine or in slight Trendelenberg position and the entry site covered by an air occlusive dressing. A satisfactory choice is a transparent semipermeable dressing like Tegaderm. ${ }^{7}$ Alternatively, a gauze dressing may be used with firm manual pressure, and once haemostasis is achieved an air occlusive dressing substituted. A gauze dressing is not air occlusive unless completely covered by tape, as illustrated by two instances of venous air embolism with sitting up some 10-15 minutes after catheter removal. ${ }^{6} \mathrm{~A}$ definitive length of time for keeping the dressing has not been determined, but intervals of 24-72 hours have been suggested.

\section{Treatment}

Successful treatment of venous air embolism relies on rapid recognition and prompt action. Further air entry must be stopped. The patient should immediately be placed in a head down, left lateral position, allowing blood to pass through the inferiorly located pulmonary outflow tract. Lowering the head may also decrease the risk of paradoxical air embolism to the brain through a patent foramen ovale. The patient should breathe $100 \%$ oxygen to promote reabsorption of nitrogen from the pulmonary circulation. If cardiac arrest occurs, then external cardiac massage may move the air embolus into the pulmonary vasculature, where capacitance is greater, thereby relieving the obstruction to right ventricular outflow. If a central venous catheter is in place, then aspiration of air should be attempted. Thoracotomy and open chest cardiac massage followed by aspiration of air directly from the right ventricle has been successful in isolated cases of massive air embolism. Hyperbaric oxygen may be of benefit. If these manoeuvres fail emergency cardiopulmonary bypass has been advocated.

electrolyte concentrations were normal. Blood gas values measured with the patient breathing $35 \%$ oxygen were oxygen tension $18.4 \mathrm{kPa}$, pressure of carbon dioxide $5 \cdot 2 \mathrm{kPa}, \mathrm{pH} 7 \cdot 27$, standard bicarbonate concentration $17.6 \mathrm{mmol} / \mathrm{l}$, and base excess $-8.4 \mathrm{mmol} / \mathrm{l}$. The electrocardiogram showed sinus 